\title{
THE LYMAN ALPHA BULGE OF JUPITER: EFFECTS OF NON-THERMAL VELOCITY FIELD
}

\author{
Lotfi Ben Jaffel ${ }^{1}$, John T. Clarke ${ }^{2}$, Renée Prangé ${ }^{3,1}$ G. Randall Gladstone', and Alfred Vidal-Madjar ${ }^{1}$
}

Abstract. In this preliminary study, we outline for the first time the effect of non-thermal line broadening processes (turbulence, random waves, convection ,...) on the shape and intensity of the $\mathrm{H} \mathrm{Ly}-\alpha$ line resonance scattered from the atmosphere of Jupiter and we show how a nonthermal velocity field, confined to the bulge region, in the upper atmosphere of Jupiter, may account for most of the $\mathrm{H} \mathrm{Ly}-\alpha$ bulge features. Both the shape and the brightness of the Ly- $\alpha$ line from the bulge region as reported by the IUE instrument and the Voyager UV Spectrometer can be recovered assuming resonant scattering with a total atomic hydrogen $[\mathrm{H}] \sim 4 \times 10^{17} \mathrm{~cm}^{-2}$, and a non-thermal component $[\mathrm{H}] \sim 2 \times 10^{15} \mathrm{~cm}^{-2}$ above the thermopause.

\section{Introduction}

A sounding rocket measurement in 1978 , and the Voyager 1 and 2 encounters with Jupiter during 1979, revealed a strong and enigmatic asymmetry, called the "bulge," in the Jovian H Ly- $\alpha$ emission (Clarke et al., 1980; Sandel et al., 1980). More than one decade of observations has shown that this is a long-lived stable feature of the Jovian airglow, characterized by a significant enhancement of the Ly- $\alpha$ emission centered on the magnetic drift equator, and fixed in magnetic longitude (system III) at $\lambda_{I I I} \simeq 100^{\circ}$ (Dessler et al., 1981; Skinner et al., 1988). The Voyager UVS data revealed a similar relative brightening on the dark side of the planet (McConnell et al., 1980). Finally, no corresponding asymmetry in the Jovian $\mathrm{H}_{2}$ Lyman and Werner band emissions has been observed (McGrath et al., 1990), and this puzzling fact puts severe constraints on the interpretation of the Ly- $\alpha$ bulge.

Recently, new data were obtained with IUE, which cast new light on this puzzling problem. It consists of Ly- $\alpha$ line profiles recorded in the Jovian equatorial region (Clarke et al., 1991) with the small aperture at high dispersion ( $0.14 \AA$ resolution). Among them are line profiles from the bulge at the limb and at the disc center (Figure 1). The lines are surprisingly broad in the core with very reduced wings. By contrast, the lines from midlatitudes and the anti-bulge region showed significantly less brightening and broadening (Figure 2).

Two possible explanations for the observed line broadening are non-thermally-enhanced Doppler motions of $\mathbf{H}$ atoms increasing their cross section at $\mathrm{Ly}-\alpha$, or an internal source of Ly- $\alpha$ within the Jovian atmosphere. In view of the day/night asymmetry in the brightness excess of the bulge region over the non bulge one (e.g. Sandel et al.,

\footnotetext{
${ }^{1}$ CNRS, Paris, France

${ }^{2}$ University of Michigan

${ }^{3}$ CNRS, Orsay, France

${ }^{4}$ University of California, Berkeley
}

Copyright 1993 by the American Geophysical Union.

Paper number $92 \mathrm{GL} 02488$

0094-8534/93/92GL-02488\$03.00
1980), and since there is no evidence for enhanced charged particle excitation of $\mathrm{H}_{2}$ along the equator as would be expected to accompany an internal source, we dismiss any collisional mechanism as the dominant process to produce the Ly- $\alpha$ bulge (except the Shemansky model for the moment), and we will therefore focus on modeling an upper atmospheric non-thermal velocity field which may explain the bulge phenomenon in terms of line profiles as well as in terms of longitudinal brightness distribution.

\section{Line profile formation}

Our radiative transfer ( $R-T)$ model is based on the discrete ordinate method (Ben Jaffel and Vidal-Madjar, 1989). We used the 16-stream approximation and the angle averaged frequency redistribution function (AAPR). First considering only resonant scattering of the solar and interplanetary Ly- $\alpha$, we computed brightnesses for a range of $\mathbf{H}$ column densities and distributions. A good fit was obtained for the off equatorial region (SWP 41480) with $[\mathrm{H}] \sim 3.7 \times 10^{17} \mathrm{~cm}^{-2}$ which is 3 to 4 times the occultationderived total column density (Figure 3). Although we could find an approximate fit to the central meridian bulge line profile with $[\mathrm{H}] \sim 10^{18} \mathrm{~cm}^{-2}$ (for any value of $[\mathrm{H}]$, the fit is always poor near the line core) (figure 1), we were unable to fit the limb bulge line profile, both in shape and brightness (figure 1), with any realistic combination of our model parameters $[H]$ (e.g. Clarke et al., 1991), $\frac{d T}{d z}$ in the thermosphere, and the exospheric temperature.

Then, considering the suggestion of a possible collisional internal Ly- $\alpha$ contribution near the exobase (Shemansky, 1985), we introduced an internal source in the way described by Ben Jaffel et al. (1991). The major effect of such an internal source is to increase the Jovian Ly- $\alpha$ brightness, the line width being comparable to or even smaller than for resonance scattering of sunlight. This could not either help to recover the line shapes shown in Figure 1. A strong contribution ( $50 \%$ or more) from an internal source of the kind proposed by Shemansky conflicts with the present IUE data as shown in Figure 2. Indeed, the intensity of the line center is nearly the same for both the bulge (SWP 38165) and mid-latitude (SWP 41480) line profiles. This would not be the case if the Shemansky electroglow model were the dominant process in the Ly $\alpha$ emission because it would produce an optically thin line that would increase the intensity at the line center. From Figures 2 and 3 , we estimate that such an internal source, if it exists, does not contribute more than $10 \%$ of the observed signal.

We then considered the possibility of a line broadening mechanism other than natural or thermal, acting on the line within the Jovian atmosphere. In view of the low particle density in the region of formation of the Ly- $\alpha$ line, broadening by collisions or the Stark effect are ineffective (by several orders of magnitude), but the effect of "turbulence" cannot be rejected a priori. The exact nature of this process is not determined in this paper, and will henceforth be described by the astrophysical term "turbulence". We also cannot determine uniquely the altitude at which this occurs. Note that a non-thermal veloci- 

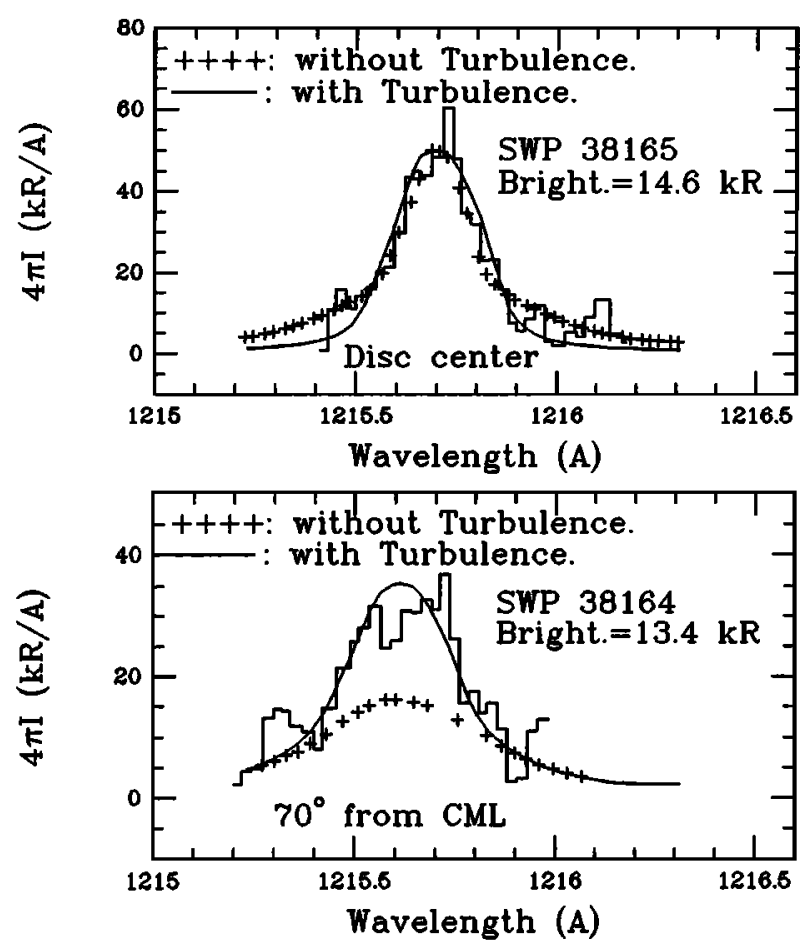

Fig. 1: H Ly $\alpha$ line profile in the bulge of Jupiter. (Upper) the bulge is close to the disc center. (Lower) The bulge is near the disc limb. Model curves have been convolved with the IUE point spread function, and integrated over the 3 arcsec projection on the Jovian disc of the IUE small aperture. Model calculation with turbulence was carried out for resonant scattering. The best fits are obtained for $[\mathrm{H}] \simeq 3.7 \times 10^{17} \mathrm{~cm}^{-2}$ and a turbulent velocity $\zeta=9 \mathrm{~km} \mathrm{~s}^{-1}$. (Upper) the model without turbulence required $[\mathrm{H}] \sim 10^{18} \mathrm{~cm}^{-2}$. (Lower) without turbulence, it is impossible to recover the line profile from the same bulge region when observed near the disc limb for any value of $[\mathrm{H}]$. The solar flux $\simeq 3.5 \times 10^{11}$ photons $\mathrm{cm}^{-2} \mathrm{~s}^{-1}$.

ty component near the thermopause would not lead to substantial dissipation of energy, whereas lower in the atmosphere, collisional dissipation could substantially alter the thermal distribution. This qualitatively constrains the total column and the altitude of the turbulent region.

Traditionally, the astrophysical definition of turbulence involves classical hydrodynamic turbulence, convective or chaotic motions. When non-thermal effects are included, the radiative transfer equation is generally

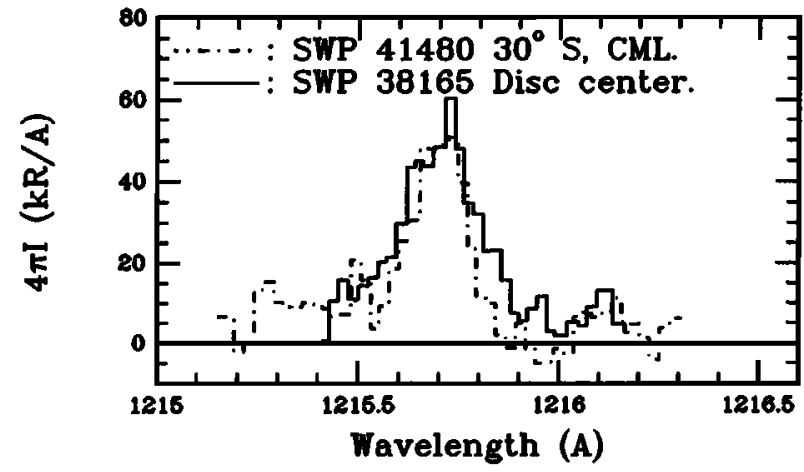

Fig. 2: H Ly- $\alpha$ line profiles in and off the bulge region.

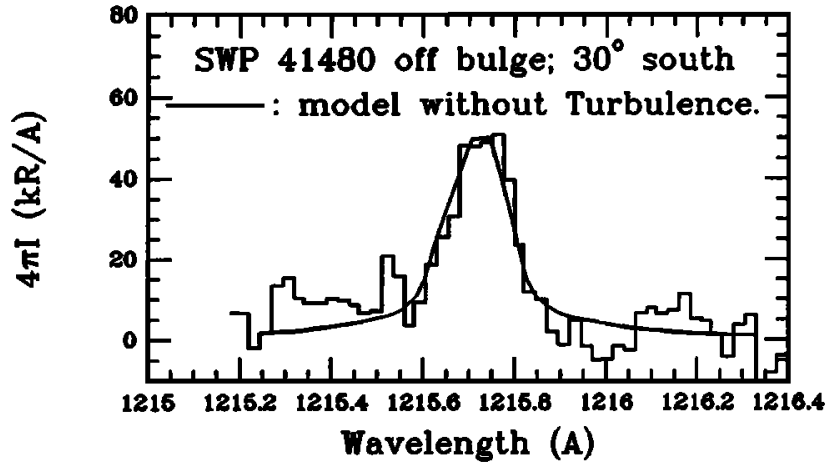

Fig. 3: The model curve has been convolved with the IUE point spread function. The calculation was carried out for resonant scattering, $[\mathrm{H}]=3.7 \times 10^{17} \mathrm{~cm}^{-2}$ and no turbulence. The solar flux $\simeq 3.5 \times 10^{11}$ photons $\mathrm{cm}^{-2} \mathrm{~s}^{-1}$.

stochastic and strongly coupled to the hydrodynamic equations describing the atmospheric state. Usually, the kinematic approximation is employed and the two problems are treated as decoupled (Cram et al., 1979). Even at this limit, the solution of the radiative transfer equation is still very difficult and not yet well defined (Magnan, 1976). However, the problem can be handled by considering the two classic small scale (micro) and large scale (macro) limits of the process.

Because the Ly- $\alpha$ line width is large, we found that macroturbulent velocities smaller than $10 \mathrm{~km} \mathrm{~s}^{-1}$ lead to no perceptible effects on the observed line shape. Considering a turbulent velocity field with a Gaussian distribution, the microturbulent approximation thus consists in defining an effective Doppler width $\Delta \nu_{D}=\frac{\nu_{u}}{c}\left(\frac{2 k_{\nu} T}{m_{H}}+\zeta^{2}\right)^{\frac{1}{2}}$ where $\zeta$ is the most probable turbulent velocity. We must emphasize that the approximation of microturbulence is just considered as a simplification to handle the difficult stochastic R-T equation and not as a constraint on the nature of the turbulent flow. We have generated resonance scattered $\mathrm{Ly}-\alpha$ profiles with a variety of velocity values, and we have been able to reproduce the shape of the IUE bulge profiles quite well. The best fit is ob-

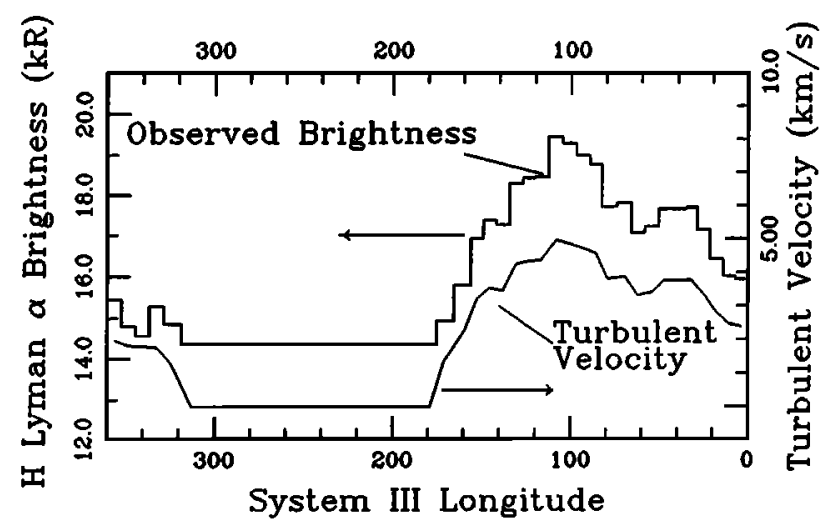

Fig. 4: Histogram: longitudinal variation of the equatorial Jupiter H Ly- $\alpha$ brightness as inferred from Voyager 2 UVS pre-encounter N-S map (Sandel et al., 1980). Curve : longitudinal variation of the turbulent velocity field needed to account for the measured brightness (the anti-bulge brightness has been approximated by its average value). $[\mathrm{H}]=3.7 \times 10^{17} \mathrm{~cm}^{-2}$, and the solar flux $\simeq 7 \times 10^{11}$ photons $\mathrm{cm}^{-2} \mathrm{~s}^{-1}$. 
tained for $\zeta=9 \mathrm{~km} \mathrm{~s}^{-1}$ (Figure 1). In this model, the non-thermal column corresponds to $[\mathrm{H}] \sim 2 \times 10^{15} \mathrm{~cm}^{-2}$ which represents less than $1 \%$ of the total [H] content. A solar Ly $\alpha$ flux of $3.5 \times 10^{11}$ photons $\mathrm{cm}^{-2} \mathrm{~s}^{-1}$ at $1 \mathrm{AU}$ is required to match the $\sim 14 \mathbf{k R}$ brightness of the emission, within $10 \%$ of the value derived by Shemansky (private communication) for the same period. This provides evidence that, at least in this example, the turbulence effects can account for the brightness of the bulge emission as well as for the broadening of the line.

To further check our results we considered a microconvective (instead of microturbulent) motion in the bulge region (She, private communication). The velocity field in this case can be described by a dual-gaussian distribution respectively centered at $\pm v_{0}$. The best fit was obtained for $v_{0}=9 \mathrm{~km} \mathrm{~s}^{-1}$ and a gaussian dispersion of $3 \mathrm{~km} \mathrm{~s}^{-1}$, which is consistent with the microturbulence model results.

As just emphasized, turbulence can increase the brightness of the emergent Ly- $\alpha$ emission. We have therefore considered the longitudinal variation of the equatorial Ly- $\alpha$ brightness derived from the Voyager 2 UVS preencounter N-S map sequence (Sandel et al., 1980). Using the same atmospheric model and the solar flux corresponding to this period, we have adjusted the microvelocity values so as to reconstruct the observed brightness asymmetry (Figure 4). The velocity field matching the observations extends in the E-W direction over 180 degrees, the peak value, $\zeta=5 \mathrm{~km} \mathrm{~s}^{-1}$ at about $\lambda_{111}=100^{\circ}$, is smaller than the velocities derived from the IUE line profiles in the bulge, and the brightness outside the bulge could be fully accounted for without any turbulence and with $[H]=3.7 \times 10^{17} \mathrm{~cm}^{-2}$.

The line profiles show that the results are also slightly sensitive to the altitude of the micro-velocity field, and suggest that the turbulence primarily affects the upper layers of the atmosphere: all the fits presented here were obtained with a constant velocity field confined to the region above the thermopause. This choice is somewhat arbitrary, but further analysis using UVS data for different wavelength ranges (i.e. He $584 A$, Lyman and Werner bands and Ly- $\beta$, Shemansky, 1985) may improve our knowledge of the gradient of the velocity field as these lines form at different levels of the atmosphere.

\section{Discussion}

A source of energy is required to drive the "turbulence". The compressibility of the atmosphere, its rapid rotation and magnetic effects could contribute to its onset, but this is a very complex non-linear problem, outside the scope of the present study. Therefore, our model is very simplified and our results should be considered qualitative. In addition, we should keep in mind that the velocities we derive for the non-thermal field are effective velocities i.e. deduced as the result of one particular scale length. Moreover, the magnitude of these effective velocities is an upper limit, since any coupling with the atmospheric hydrodynamics has been neglected in this preliminary study. On the other hand, the derived velocities, which are supersonic, may favor the development of shock waves.

The strength of the required energy source can be estimated as follows. Without any loss of generality the input energy can be considered as completely dissipated into heat in the upper atmosphere of Jupiter. From Bachelor (1953), we define $\epsilon=\mu \frac{\zeta^{2}}{l^{2}}$ as the dissipation rate per mass unit, where $L$ is the scale length of the eddy responsible for the turbulence and $\mu \simeq 10^{-4} \mathrm{~g} \mathrm{~cm}^{-3} \mathrm{~s}^{-1}$ is the dynamic viscosity of $\mathrm{H}_{2}$. The problem now is to define the scale length $L$. One possibility is to consider $L$ as the mean free path of a Ly- $\alpha$ photon in the expected turbulent region. In the case of a strong line (as is the case here), the line optical depth $\tau_{l} \simeq 1$ is frequency dependent and should be defined for the frequency range corresponding to the part of the line that is expected to be affected by the random velocity field. From Figure 2, we can see that the major effect of the non-thermal velocity field is to broaden the line core. Usually, a line core is defined by a frequency range in Doppler units between the line center and $x_{c} \simeq 3$. For an atomic H density $n_{H} \simeq 5 \times 10^{7} \mathrm{~cm}^{-3}$, a Voigt function as the $H$ absorption profile at $\mathrm{Ly}-\alpha$, and $\tau_{l} \simeq 1$ in this frequency range, the turbulence scale length must be smaller than $3 \times 10^{5} \mathrm{~km}$. Since this upper limit is larger than Jupiter, it does not provide a practical limit to the actual scale length. Furthermore, the IUE data in Figure 2 shows that the line center frequency cannot in this case represent the line broadening as the corresponding intensity is the same for the bulge and the off bulge regions.

Assuming that the random velocity field is confined within the upper atmosphere of Jupiter, around the thermopause, we then adopt the atomic $H$ scale height to be a scale length for the turbulent flow: this choice has the advantage that it both defines the vertical scale of the turbulent region and is in the range of the scale lengths related to the line core definition. This scale length provides a good fit to the observed line profiles. It also describes the turbulent motion of a vertical molecular hydrogen density column of about $1.8 \times 10^{17} \mathrm{~cm}^{-2}$ (and atomic hydrogen density column of about $\left.2 \times 10^{15} \mathrm{~cm}^{-2}\right)$ in the upper part of the Jovian atmosphere.

Thus, taking $L \simeq 380 \mathrm{~km}$ for the non-thermal region with a turbulent velocity $\zeta \simeq 9 \mathrm{~km} / \mathrm{s}$, we obtain a dissipation rate $\epsilon \simeq 5.6 \times 10^{-8} \mathrm{erg} \mathrm{cm}^{-3} \mathrm{~s}^{-1}$. Integrated over the $H$ vertical scale height, this leads to a peak dissipation flux $\bar{\epsilon} \simeq 2.1 \mathrm{erg} \mathrm{cm}^{-2} \mathrm{~s}^{-1}$. These rates should be lowered to $\sim 1.1$ ergs $\mathrm{cm}^{-2} \mathrm{~s}^{-1}$ of the same order of magnitude as the values $\left(\sim 0.4 \mathrm{ergs} \mathrm{cm}^{-2} \mathrm{~s}^{-1}\right)$ inferred from Voyager UVS observations (Broadfoot et al., 1981), when considering the longitudinal non-uniformity of the turbulent velocity field shown in Figure 5.

Thus, if turbulence is confirmed, it may also offer an explanation for the heating excess observed for Jupiter (Atreya et al., 1979). The maximum energy necessary to sustain the turbulent flow $\left(\sim 2.1\right.$ ergs $\left.\mathrm{cm}^{-2} \mathrm{~s}^{-1}\right)$ is fairly large for a planetary atmosphere. However, it is known that a huge energy from magnetospheric particle precipitation $\left(10 \sim 20 \mathrm{ergs} \mathrm{cm} \mathrm{cm}^{-2} \mathrm{~s}^{-1}\right.$ depending on the size of the auroral UV oval) is injected at auroral latitudes. A mechanism related to the auroral energy could provide the turbulence energy. Indeed, this is consistent with some indications of mass transport $(20 \sim 40 \mathrm{~km} / \mathrm{s})$ in the auroral regions observed in the Jovian auroral Ly- $\alpha$ line (Clarke et al., 1989) although such a coupling has to be defined. In the long-term, the $\mathrm{H} \mathrm{Ly- \alpha}$ intensities from both bulge and anti-bulge regions follow, on the average, the solar $H$ Ly- $\alpha$ flux, indicating that both emissions could be due to resonance scattering of the solar flux in a quasi-stationary atmosphere. However, Skinner et al. (1988) give evidence of some extra variability of the bulge brightness within a factor of \pm 2 including a variation of a factor 1.3 once observed within only two days. In that case, the observed short-term behavior could be due to short term variations of the turbulence in the bulge around an average value, reflecting similar variations in the process responsible for the turbulence. Indeed, temporal variations in auroral particle precipitations are typically of the order of a factor of 2 to 3 including variation of few days (Prangé, 1992) to a few hours ( Livengood et al., 1991).

The presence of the Ly- $\alpha$ bulge on the nightside of the planet is also consistent with our interpretation. The 
main difference from the dayside is that the source of external Ly- $\alpha$ photons is himited to the interplanetary wind scattering. With the same atmospheric model and the velocity distribution derived from the analysis of the dayside bulge emission we were indeed able to reproduce, given the $\sim 700 \mathrm{R}$ recorded from the non-bulge region, the $\sim 1000 \mathrm{R}$ nightside bulge brightness reported by McConnell et al. (1980). It follows that the spatial distribution of the turbulent velocity field should be fixed in the longitude system III (e.g. Figure 4) and independent of the Jovian local time.

The longitudinal uniformity of the $\mathrm{H}_{2}$ bands is consistent with our turbulence model, regardless of which of the two proposed mechanisms, fluorescence or electroglow, excites them. Fluorescence operates principally near the homopause, where the turbulence can be too weak to broaden the $\mathrm{H}_{2}$ emission lines significantly. Electroglow is claimed to originate near the exobase (Shemansky, 1985), a region of strong turbulence, but the e- $\mathrm{H}_{2}$ collision cross section is insensitive to turbulence. However, as inferred from the IUE data (see Figures 2 and 3), the electroglow contribution to the Jovian Ly- $\alpha$ emission is less than $10 \%$ as previously discussed. This new result casts doubts on the ability of the electroglow mechanism, as presented by

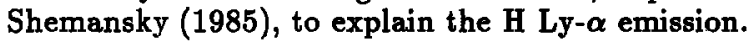

All the calculations were performed for resonant scattering as the dominant process and omitted any minor contribution from uniform charged particle excitation (other than Shemansky model). It is beyond the scope of this paper to model quantitatively the contribution of the different mechanisms to the total Jovian Ly- $\alpha$ emission.

\section{Conclusion}

From modeling the radiative transfer properties needed to reproduce the observed broad Ly- $\alpha$ emission line profiles from Jupiter's equator, we propose the existence of a turbulent layer of the Jovian atmosphere of $\sim 2 \times 10^{15} \mathrm{~cm}^{-2} \mathrm{H}$ atoms embedded in a region where the total vertical atomic hydrogen column is $3.7 \times 10^{17} \mathrm{~cm}^{-2}$. We find that non-thermal velocity field of a few $\mathrm{km} / \mathrm{s}$ is consistent with the following characteristics of the observed Jovian Ly- $\alpha$ equatorial airglow:

- very broad Ly- $\alpha$ line core in the bulge,

- short term and long term variations,

- longitudinal brightness asymmetry fixed in magnetic field (bulge),

- day/night asymmetry preserving the bulge,

- lack of bulge in the $\mathrm{H}_{2}$ emissions,

- heating excess of the Jovian upper atmosphere.

This mechanism, in contrast to previous ones (Sandel et al., 1980, Dessler et al., 1981), does not require large scale changes in the Jovian equatorial atmospheric composition. We have developed a preliminary simplified radiative transfer model that includes the effect of the nonthermal velocity field, and we have been able to fit the observed line profiles in the bulge, as well as the longitudinal brightness distribution. The origin of this velocity field may be related to the Jovian aurora given the supersonic blue shift recorded for the Ly- $\alpha$ auroral line and the huge energy injected at high latitude by magnetospheric particles. However, the nature of this velocity field, along with hydrodynamic and shock wave effects on the airglow of Jupiter have not yet been well established, and are under investigation.

Acknowledgements. We are grateful to B.R. Sandel, Z. She, D.E. Shemansky, and R.V. Yelle for very helpful discussions since the first submission of this paper in april 1991. This work was supported by CNRS (France), and NASA Grants NAGW-1766 and NAG5-1030 to the University of Michigan.

\section{References}

Atreya, S.K., et al., Jovian upper atmospheric temperature measurements by the Voyager 1 UV Spectrometer, Geophys. Res, Lett., 6, 795, 1979.

Bachelor, G.K., The theory of homogeneous turbulence, Cambridge Univ. Press, 1953.

Ben Jaffel, L. and A. Vidal-Madjar, The Lyman alpha albedo of Jupiter , Astron. Astrophys., 220, 306, 1989.

Ben Jaffel, L., et al., A model for the disc Lyman alpha emission of Uranus, J. Geophys. Res., 96, 7296, 1991.

Broadfoot, L., et al., Overview of the Voyager UVS results through Jupiter encounter, J. Geophys. Res., 86, 8259, 1981.

Clarke, J.T., et al., Spatial imaging of hydrogen Ly-a emission, Astrophys. J., 240, 696, 1980.

Clarke, J.T., J. Trauger, and J.H. Waite, Doppler shifted H Ly- $\alpha$ emission from Jupiter's Aurora, Geophys. Res. Lett., 16, 587, 1989.

Clarke, J.T., R.G. Gladstone, and L. Ben Jaffel, Jupiter's dayglow H Ly- $\alpha$ emission line profile, Geophys. Res. Lett., 18,1935, 1991.

Cram, L.E., S.L. Keil, and P. Ulmschneider, Some effects of acoustic waves on spectral line profiles, Astrophys. J, 234, 768, 1979.

Dessler, A.J., B.R. Sandel, and S.K. Atreya, The Jovian hydrogen bulge: evidence for corotating magnetospheric convection, Planet. Space Sci, 29, 215, 1981.

Livengood, T.A., R. Prangé, G.E. Ballester, and H.W. Moos, Exceptional variability of the Jovian UV aurora of december 1990, EOS, 72, 186, 1991.

Magnan, C., An approximation for solving the non-LTE line transfer in a spatially correlated random velocity field, J. Quan. Spectrosc. Radiat. Transfer, 16, 281, 1976.

McConnell, J.C., B.R. Sandel, and A.L. Broadfoot, Airglow from Jupiter's nightside and crescent: UVS observations from Voyager 2, Icarus, 43, 128, 1980.

McGrath, M., G.E. Ballester, and H.W. Moos, Jovian $\mathrm{H}_{2}$ dayglow emission (1978-1989), J. Geophys. Res., 95, $10,365,1990$.

Prangé, R., The UV and IR Jovian Aurorae, Adv. Space Res., 12, (8)379, 1992.

Sandel, B.R., A.L. Broadfoot, and D.F. Strobel, Discovery of a longitudinal asymmetry in the hydrogen Lymanalpha brightness of Jupiter, Geophys. Res. Lett., 7, 5, 1980.

Shemansky, D.E., An explanation for the H Ly- $\alpha$ longitudinal asymmetry in the equatorial spectrum of Jupiter, J. Geophys, Res., 90, 2673, 1985.

Skinner, T.E., et al., Temporal variation of the Jovian H I Lyman alpha emission (1979-1986), J. Geophys. Res., 93, 29, 1988.

${ }^{1}$ Lotfi Ben Jaffel and Alfred Vidal-Madjar, Institut d'Astrophysique de Paris, 98bis Bd Arago, 75014 Paris, France.

${ }^{2}$ John T. Clarke, Department of Atmospheric, Oceanic, and Space Sciences, The University of Michigan, Ann Arbor MI 48109-2143.

${ }^{3}$ Renée Prangé, Institut d'Astrophysique Spatiale, University Paris XI, Bat. 121, 91405 Orsay, France.

${ }^{4}$ G. Randall Gladstone, Space Sciences Laboratory, University of California, Berkeley CA 94720.

(Received: January 6, 1992;

Revised: July 30, 1992;

Accepted: September 30, 1992) 